\title{
Whole Blood Assay to Access T Cell-immune Responses to Mycobacterium tuberculosis Antigens in Healthy Brazilian Individuals
}

\author{
Paulo RZ Antas, Fernando LL Cardoso, Eliane B Oliveira, Patrícia KC Gomes, \\ Kátia S Cunha*, Euzenir N Sarno, Elizabeth P Sampaio/ ${ }^{+}$
}

Laboratório de Hanseníase, Instituto Oswaldo Cruz-Fiocruz, Av. Brasil 4365, 21045-900 Rio de Janeiro, RJ, Brasil

*Hospital Municipal Raphael de Paula e Souza, Rio de Janeiro, RJ, Brasil

The production of interferon $\gamma(I F N \gamma)$ guarantees effective T cell-mediated immunity against Mycobacterium tuberculosis infection. In the present study, we simply compare the in vitro immune responses to Mycobacterium antigens in terms of IFN $\gamma$ production in a total of 10 healthy Brazilian volunteers. Whole blood and mononuclear cells were cultivated in parallel with PPD, Ag85B, and M. bovis hsp65, and five-days supernatants were harvested for cytokine detection by ELISA. The inter-assay result was that the overall profile of agreement in response to antigens was highly correlated $\left(\mathrm{r}^{2}=0.9266 ; \mathrm{p}=0.0102\right)$. Potential analysis is in current progress to dictate the usefulness of this method to access the immune responses also in tuberculosis patients and its contacts.

Key words: tuberculosis (TB) - T cell-responses - whole blood - PPD - latent TB infection - interferon $\gamma$

Tuberculosis (TB) remains the main infectious health care problem worldwide with a total of 8 million new cases developing each year (Kochi 1991). The importance of T cells in the protective immune response against $\mathrm{Myco}$ bacterium tuberculosis (Mtb) has long been well-known since their major function is the production of interferon $\gamma(\mathrm{IFN} \gamma)$ and tumor necrosis factor (TNF $\alpha)$, which have been verified to play a crucial role in macrophage activation, control of mycobacteria replication, and granuloma formation both in humans and mice (reviewed by Stenger \& Modlin 1999).

Assessment of $\mathrm{T}$ cell function in response to recombinant Mtb antigens has been performed in an endemic TB area in Brazil. In previous studies, we have shown that IFN $\gamma$ response in the TB patient group was enhanced in comparison to that of the controls, and the CD69 and CD25 in vitro expression on T cells in response to Mtb Antigen 85B (Ag85B) and Ferritin was enhanced after stimulation (Cardoso et al. 2002, Antas et al. 2002).

Here, a six-month comparative study in terms of IFN $\gamma$ production was performed between whole blood assay (WBA) and mononuclear cells (PBMC) cultivated in parallel in the presence of protein purified derivative (PPD), Ag85B, and heat-shock protein $M$. bovis hsp65 among a total of 10 healthy, Rio de Janeiro city residents for the purpose of validating the WBA methodology in the study of in vitro immune response to Mtb antigens. Complete information of the cohort, Mantoux test performed and studied area is described elsewhere (Cardoso et al. 2002).

Financial support: $\mathrm{CNPq}$ and Fiocruz

${ }^{+}$Corresponding author. Fax: +55-21-2270.9997. E-mail: esampaio@ioc.fiocruz.br

Received 4 April 2003

Accepted 19 November 2003
For blood collection, PPD-negative individuals [5 males and 5 females; mean age $( \pm S D)$ of $23 \pm 2$, ranging from 21 to 25] with no knowledge of prior contact or history of TB disease, voluntarily provided written consent as approved by Fiocruz Ethics Review Committee (Resolution 196/96 National Health Council). Briefly, heparinized venous blood was drawn and for the WBA, a 1:3 dilution with RPMI 1640 medium supplemented with $20 \%$ autologous plasma (only for PBMC), $100 \mathrm{U} / \mathrm{ml}$ penicillin, $100 \mu \mathrm{g} / \mathrm{ml}$ streptomycin, and 2mM L-glutamine (Gibco BRL) was seeded in 96-well round-bottom plates (Costar Corporation, Cambridge, MA) at $200 \mu \mathrm{l} /$ well. For PBMC, cells were isolated by Ficoll-Hypaque (Pharmacia Fine Chemicals, Piscataway, NJ) density centrifugation. A total of $2 \mathrm{x}$ $10^{5}$ cells/well were cultivated at $37^{\circ} \mathrm{C}$ in $200 \mathrm{ml}$ of complete culture medium. All antigens used were negative for endotoxin contamination (Limulus amoebocyte lysate assay kit QCL-1000, Biowhittaker, CA). The antigens were kindly provided by Drs T Ottenhoff, K Franken, and $\mathrm{P}$ Klatser (The Netherlands), and purification methodology is detailed elsewhere (Franken et al. 2000). PPD was purchased from the Statens Serum Institute (Copenhagen, Denmark). After previous titration, the final concentration was $5 \mu \mathrm{g} / \mathrm{ml}$ for antigens, and $1 \%$ for the mitogen Phytohemagglutinin (PHA, Gibco BRL). Antigen or mitogen was added to the wells in triplicates; and after 5 days, supernatants from each assay were harvested, respectively pooled and kept at $-20^{\circ} \mathrm{C}$ until further use. Control wells were comprised of cells cultivated in medium alone. Concentration of IFN $\gamma$ in cell-free culture supernatants was determined by using a commercially specific enzymelinked immunosorbent assay (ELISA) processed according to the manufacturer's specifications (Pharmingen Inc., San Diego, CA). Cytokine levels were expressed as pg/ $\mathrm{ml}$, and the detection limit of the assay was $8 \mathrm{pg} / \mathrm{ml}$. More detailed methodology is described elsewhere (Cardoso et al. 2002). Data were reported as mean \pm standard error of mean (SEM), the brackets indicate lower to higher quartiles, 
and student's $t$ tests and linear regression were used to perform statistical analysis (GraphPad InStat V. 2.04), after setting the significance level $p \leq 0.05$.

As a positive control, PHA-stimulated cultures were used as comparison, and positive responses (IFN $\gamma>100$ $\mathrm{pg} / \mathrm{ml}$ ) were equally detected in all but one individual tested (for WBA $=4,752 \pm 1,391 \mathrm{pg} / \mathrm{ml}[1,544.4$ to $7,959.6]$ and $\mathrm{PBMC}=4,899 \pm 1,398 \mathrm{pg} / \mathrm{ml}[1,675.2$ to $8,122.8])$. In general, the comparisons of induced IFN $\gamma$ levels with Mtb antigens were very similar in both methods $\left(r^{2}=0.9266\right.$, $p=0.0102$, Figure). The WBA vs PBMC variances are also respectively represented: [PPD $=729.7$ to $1,236.3 \mathrm{vs}$ 661.8 to $1,218.2 ; \mathrm{Ag} 85 \mathrm{~B}=381.3$ to 928.7 vs 294.8 to $1,041.2$; hsp $65=46.5$ to 369.5 vs 183.2 to 618.8 ; baseline $=0.1$ to 126.5 vs 2.8 to 57.1]. Although enhanced IFN $\gamma$ levels, no difference was observed when $M$. bovis hsp65-stimulated PBMC was compared to WBA $(p=0.15)$. Likewise, the baseline levels were also equivalent between the methods (Figure). Extra care was taken to make sure the data were as precise as possible. As such, two antigen concentrations were used with similar results (data not shown).

Our major goal here was to test a reliable, reproducing, fast and easy in vitro method to access the T cell immune responses. In addition, by using WBA a more physiological environment is maintained, which may have an effect on the immune response to the antigenic stimulation. A choice was made to detect IFN $\gamma$ by ELISA as useful to induce response by well-defined Mtb antigens, and as described before, positive IFN $\gamma$ levels for Ag85B

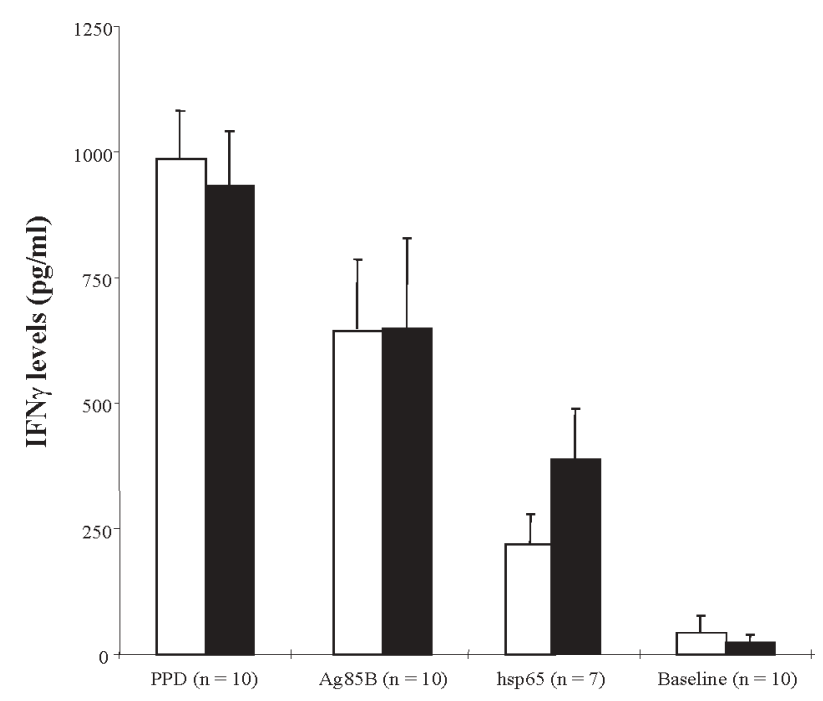

\section{Stimulus}

Interferon $\gamma($ IFN $\gamma)$ production $(\mathrm{pg} / \mathrm{ml})$ evaluated by specific ELISA in response to protein purified derivative (PPD), Mycobacterium tuberculosis Antigen 85B (Ag85B), and M. bovis hsp65 comparing whole blood assay (WBA, white bars) and mononuclear cells (PBMC, black bars) methods in PPD-negative healthy individuals. For positive control of cytokine secretion in culture supernatants, Phytohemagglutinin (PHA)-stimulated cultures were used $(n=9$, see text), and for negative control, the baseline levels were also represented. Bars represent the mean (+ SEM) and $\mathrm{n}$ express number of individuals. Using the cut off of IFN $\gamma>100 \mathrm{pg} / \mathrm{ml}$, paralleled positive responses were detected in most of individuals tested by both methods. were also confirmed (Cardoso et al. 2002). For the first time, WBA vs PBMC from healthy controls in Brazil were used also to access the potential latent TB infection (LTBI). Formerly, it has been proposed by the Quantiferon ${ }^{\circledR}$ assay employing Mtb antigens as a tool discriminating non-symptomatic LTBI (Brock et al. 2001, Mazurek et al. 2001). Overall, there was a little inter-assays variation, even though a trend, although non-statistically significant, to higher IFN $\gamma$ levels in M. bovis hsp65-stimulated PBMC. This may reflect an immune response to BCG vaccine, revealed by an enhanced number of specific IFN $\gamma$-secreting T cells in the PBMC. The aforementioned and other studies (Cardoso et al. 2002) have found elevated IFN $\gamma$ titers in PPD-positive, healthy control individuals, as can be expected from a co-endemic TB and leprosy area. Surprisingly, the PPD-negative individuals tested herein also showed positive IFN $\gamma$ levels for all antigens tested in both methods, but mainly to PPD itself (almost 10-fold the cut off). It has been described before in another cohort (Cardoso et al. 2002), and overall sensitivity differences between both in vitro and skin tests have been observed in leishmaniasis also (DeLuca et al. 2003). That fact may support the idea that LTBI could be better detected by in vitro tests, due to its improved sensitivity, and/or that there might also be a cause of crossreactions against antigens found in other Mycobacterium species (Geluk et al. 2002). In conclusion, both in vitro methods used herein were highly correlated when accessed for the responses to Mtb and PHA antigens, and this is in keeping with another comparative study (Bocchieri et al. 1995). Now, we plan to use this method in patients for the study of potentially powerful new vaccine candidates against and diagnostic tools for TB.

\section{REFERENCES}

Antas PRZ, Oliveira EB, Milagres AS, Franken KC, Ottenhoff THM, Klatser P, Sarno EN, Sampaio EP 2002. Kinetics of T Cell-activation molecules in response to Mycobacterium tuberculosis antigens. Mem Inst Oswaldo Cruz 97: 10971099.

Bocchieri MH, Talle MA, Maltese LM, Ragucci IR, Hwang CC, Goldstein G 1995. Whole blood culture for measuring mitogen induced $\mathrm{T}$ cell proliferation provides superior correlations with disease state and $\mathrm{T}$ cell phenotype in asymptomatic HIV-infected subjects. J Immunol Methods 181: 233-243.

Brock I, Munk ME, Kok-Jensen A, Andersen P 2001. Performance of whole blood IFN-gamma test for tuberculosis diagnosis based on PPD or the specific antigens ESAT-6 and CFP-10. Int J Tuberc Lung Dis 5: 462-467.

Cardoso FLL, Antas PRZ, Milagres AS, Geluk A, Franken KLC, Oliveira EB, Teixeira HC, Nogueira SA, Sarno EN, Klatser P, Ottenhoff THM, Sampaio EP 2002. T cell responses to the Mycobacterium tuberculosis specific antigen ESAT-6 in Brazilian tuberculosis patients. Infect Immun 70: 6707-6714.

De Luca PM, Mayrink W, Santiago MA, Nogueira R, ConceiçãoSilva F, Melo G, Mendonça SCF 2003. Randomised, doubleblind, placebo-controlled study on the immunogenicity of the leishmanin skin test. Trans R Soc Trop Med Hyg 97 (in press).

Franken KL, Hiemstra HS, van Meijgaarden KE, Subronto Y, den Hartigh J, Ottenhoff TH, Drijfhout JW 2000. Purification of his-tagged proteins by immobilized chelate affinity 
chromatography: the benefits from the use of organic solvent. Protein Expr Purif 18: 95-99.

Geluk A, van Meijgaarden KE, Franken KL, Subronto YW, Wieles B, Arend SM, Sampaio EP, de Boer T, Faber WR, Naafs B, Ottenhoff TH 2002. Identification and characterization of the ESAT-6 homologue of Mycobacterium leprae and T-cell cross-reactivity with Mycobacterium tuberculosis. Infect Immun 70: 2544-2548.

Kochi A 1991. The global tuberculosis situation and the new control strategy of the World Health Organization. Tubercle 72: 71 .

Mazurek GH, LoBue PA, Daley CL, Bernardo J, Lardizabal AA, Bishai WR, Iademarco MF, Rothel JS 2001. Comparison of a whole-blood interferon gamma assay with tuberculin skin testing for detecting latent Mycobacterium tuberculosis infection. JAMA 286: 1740-1747.

Stenger S, Modlin RL 1999. T cell mediated immunity to Mycobacterium tuberculosis. Curr Opin Microbiol 2: 89-93. 